\title{
Altérité et inclusion. Du corps propre au corps social en classe de langue : emprunts à la danse contemporaine
}

Marie-Paule MULLER-JAECKI, ESPE de l'Académie de Nantes - Université de Nantes

Il faudrait inventer un temps particulier pour l'apprentissage. Le présent d'incarnation, par exemple. Je suis ici, dans cette classe, et je comprends, enfin ! Ça y est ! Mon cerveau diffuse dans mon corps : ça s'incarne.

Quand ce n'est pas le cas, quand je n'y comprends rien, je me délite sur place, je me désintègre dans ce temps qui ne passe pas, je tombe en poussière et le moindre souffle m'éparpille. (Pennac 72)

C'est en ces termes qu'un ancien cancre, devenu écrivain, nous rappelle qu'apprendre passe avant tout par le corps. «Le corps sait des choses que la tête ne sait pas encore. » (Lecoq 22). Quelle souffrance pour tout élève qui passe des années de sa vie assis, de préférence sans bouger, sur les bancs de l'école s'il ne peut pas faire corps avec les savoirs enseignés! Emprisonné dans un espace-temps aux codes inaccessibles, il assiste à son délitement en tant que sujet, à une forme de mort de son corps propre, exclu d'un corps social auquel il est censé appartenir. Physiquement présent et pourtant absent, il sent, impuissant, s'agrandir de jour en jour cette «déchirure entre soi et soi » (Cyrulnik 51) à l'origine du mécanisme d'adaptation destructeur pour la personne que les psychologues appellent «clivage ». Selon B. Cyrulnik, « la déchirure entre ce que je suis et ce que j'aspire à être constitue une véritable blessure traumatique»(31). Et l'auteur d'expliciter : «Une personnalité blessée s'adapte en se clivant : une partie sociable qui mène au succès social et l'autre partie qui meurt de honte, sans cesse, chaque jour, au moindre événement qui réveille la représentation insupportable » (63).

L'école française, peu préoccupée de ce que ressent l'élève dans son corps lorsqu'il est en apprentissage, place-t-elle ce dernier en situation de handicap ? La classe de langue étrangère, par le bouleversement identitaire qu'elle présuppose, génère-t-elle chez l'élève des perceptions qu'il vit comme particulièrement handicapantes ? «Je est un autre » en classe de langue, pour reprendre les termes d'Arthur Rimbaud: accéder à une autre langue-culture présuppose d'accepter une transformation de son corps propre pour qu'il devienne membre d'un autre corps social. Comment, pour l'enseignant de langue étrangère, amener l'élève à 
devenir autre sans qu'il se sente exclu ? Comment construire une dynamique inclusive en classe de langue?

Les éclairages ci-après proviennent de deux champs qui peuvent nous apporter des réponses : celui de la danse contemporaine et celui de l'ASH ${ }^{1}$.

La danse contemporaine s'ouvre, au travers de travaux tels ceux de la chorégraphe angevine Marie-France Roy, à des expériences très riches humainement : la création de performances $^{2}$ par des groupes incluant des danseurs trisomiques ou en situation de handicap moteur $^{3}$. L'ASH amène par ailleurs à cerner le concept d'inclusion, qui renvoie à une vision sociétale et éducative sous-tendant la loi du 11 février 2005 concernant la scolarisation des élèves en situation de handicap.

Ce qui suit vise donc à explorer la relation entre les concepts de performance et d'inclusion et ce qui fait lien avec la perspective « actionnelle » du Cadre Européen Commun de Référence pour les Langues (CECRL).

Nous nous pencherons d'abord sur le concept de performance en danse contemporaine pour voir en quoi il rencontre celui d'inclusion. Puis nous nous intéresserons plus spécifiquement à l'utilisation pédagogique de l'écriture chorégraphique pour favoriser une démarche d'inclusion en danse. Nous explorerons enfin les possibilités de transfert vers la classe de langue étrangère. Face à la complexité des interactions posées par le CECRL, est-il possible de concevoir des espaces d'apprentissage où chaque élève se sente compétent par sa contribution singulière à l'action collective ?

\section{Qu'est-ce qu'une performance?}

Selon R. Schechner, les performances sont des actions (22). Montrer l'agir, c'est être dans la performance. Tout comme le langage verbal, la performance, sans doute aussi ancienne que l'humanité, découle d'un besoin et d'une capacité de l'être humain à exprimer et partager des significations en incarnant des textes d'action (De Marinis, in Schechner 193) reliant les individus à l'intérieur d'une culture commune. Une performance peut être présentée et re-présentée.

Comme le montre Turner (Schechner 13), une performance résulte d'une tension dialectique entre le mouvement spontané constitutif de l'action d'un sujet singulier et la réflexivité qui fonde les traits communs d'une culture, ses significations, valeurs et visées partagées. 
En cela, la performance peut être considérée comme une réponse de l'être humain à la complexité du monde dans et avec lequel il interagit. Ritualisée, elle procède de codes convenus et permet la mémoire collective. Elle contribue à l' « homéostasie socioculturelle » dont parle A. Damasio (354), maintenant un équilibre dans le processus vital des individus et des groupes. Elle concourt à leur bien-être. En même temps, liée à l'ici et maintenant du sujet qui l'incarne, elle procède d'une création sans cesse rejouée dans un besoin de faire naitre et de prendre en compte l'événement singulier, imprévu, qui transforme le cours des choses.

\section{Le processus de création d'une performance en danse contemporaine}

Bien des performances en danse contemporaine sont des créations collectives auxquelles chaque danseur contribue, apportant idées et matériaux hétérogènes. Elles résultent d'un processus relativement constant, structuré autour de cinq étapes (Perez et Thomas 48) :

1. Mise en jeu de la créativité par des inducteurs. A partir de déclencheurs (objets, phrases, matières, éléments musicaux émanant du chorégraphe et/ou des danseurs euxmêmes), chaque danseur traduit en mouvement ce que ceci évoque pour lui, dans son propre imaginaire. Ce travail peut s'effectuer seul ou en petits groupes.

2. Improvisation / exploration. L'énonciation spontanée est approfondie ; le champ des possibles est exploré, seul ou à plusieurs. L'intuition et l'imaginaire sont les moteurs de la créativité. Le chorégraphe encourage la prise de risques.

3. Composition. Le chorégraphe qui a observé les improvisations, impulse une mise en cohérence qui se construit au sein du groupe selon des procédés d'écriture (variations sur un thème, combinaison aléatoire, couplet-refrain, lignes dans l'espace, alternance collectif-individuel...). La mise en synergie des propositions singulières qui se percutent et se transforment s'opère à la manière d'un collage mouvant.

4. Production. Le texte d'action est finalisé en tant qu'acte de communication entre danseurs et spectateurs. «Le fait de communiquer par le geste implique l'autre dans son corps même. D'auditeur, il devient agent; de spectateur, il devient acteur. [...] Le geste n'est pas seulement mouvement, il est fondateur de la relation avec autrui. Mieux qu'un mot, il simule une action, un acte. Il peut être arrêté ; il est signe, symbole; il est immédiatement compréhensible ; il déclenche un effet 'miroir'4" (Berthoz $126 ; 124)$.

5. Présentation. La performance qui en résulte, à chaque re-présentation, génère chez le danseur comme chez le spectateur ce ressenti de partage corps à corps qui en fait la puissance sociale. 
L'ensemble du processus créatif, savant équilibre entre une dynamique «bottom-up » et une dynamique «top-down $»^{5}$, est rendu possible par la prééminence d'une démarche «bottomup » qui donne toute sa place à la créativité singulière.

\section{Performance et handicap : les lignes de force d'une dynamique inclusive}

Pour accompagner la création d'une performance incluant des danseurs en situation de handicap, le chorégraphe procède de la même manière. En partant de la capacité d'improvisation de chaque danseur, avec ses possibilités et ses limites, il se donne un espace d'observation permettant de recueillir les propositions singulières. L'art sera de les sublimer dans une combinatoire esthétique valorisant le divers de telle sorte que, de ce processus de composition, jaillisse l'œuvre collective : «La chorégraphie ainsi ne se développera pas selon un plan, mais dans un jeu d'échanges, un jeu de possibles gestuels relié à des expériences personnelles [...] Les idées surgissent de la présence des danseurs » (Crémézi, 63-64).

Ainsi, chaque corps propre dans sa singularité contribue à former le corps social, riche de son hétérogénéité. Nous touchons là ce qui fait le cœur d'une vision sociétale et éducative inclusive. Paula Kluth la définit en ces termes : "L'inclusion est plus qu'un ensemble de stratégies ou de pratiques pédagogiques, c'est une orientation éducative qui englobe les différences et valorise le caractère unique de l'apport de chaque apprenant à l'univers de la classe $»^{6}$.

Valoriser le caractère unique de chaque contribution à la création collective est bien ce qui sous-tend à la fois la performance en danse contemporaine et une démarche inclusive.

\section{L'évolution de l'écriture chorégraphique : de l'interprétation à l'improvisation}

En quoi la danse contemporaine fait-elle rupture avec des formes plus anciennes de performance ? L'écriture chorégraphique nous aide à le comprendre.

$\mathrm{Au}$ seizième siècle en Italie sont apparus les premiers systèmes graphiques de transcription du mouvement, permettant de noter et de transmettre des performances en tant que textes d'action. Le code le plus utilisé de nos jours est la cinétographie Laban, du nom de son concepteur (1879-1958), chorégraphe et théoricien du mouvement. L'alphabet Laban est fait de symboles abstraits, pour certains en relation iconique avec le geste signifié, qui s'organisent séquentiellement de part et d'autre d'une ligne verticale représentant l'axe de gravité du corps. La partition se lit de bas en haut et permet de noter tout mouvement de n'importe quelle partie du corps. 


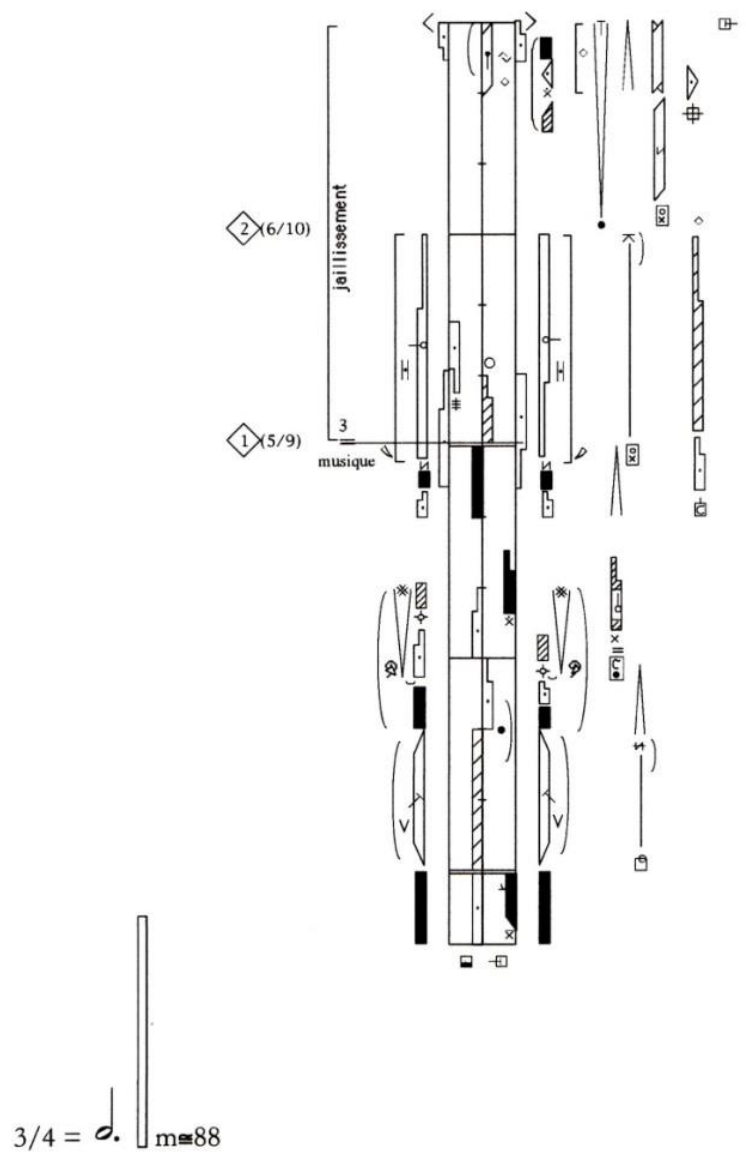

Exemple de partition chorégraphique en cinétographie Laban (Bodak 137). Chorégraphie de Malkovsky (1925) sur la musique de la $8^{\mathrm{e}}$ danse slave de Dvorak. Notation de Karin Hermès-Sunke selon l'interprétation de Suzanne Bodak.

Le lecteur accède à des «séquences sensorielles articulées capables de produire un monde. Un monde, c'est-à-dire une figure des choses, et de soi parmi les choses » (Hecquet et Prokhoris 44). Le signe appelle un geste avec une relative précision, mais « la matière de l'écriture, $[\ldots]$ ce n'est pas le plein d'une action ou d'un mouvement par avance réglés, c'est ce que l'entre-deux signes ouvre comme vacance signifiante : un appel à interpréter » (93). Interpréter, c'est faire l'intermédiaire entre deux mondes, ici, celui du texte d'action écrit qui appartient à la mémoire collective et son incarnation singulière lors de la performance dansée, re-présentée dans un ici et maintenant toujours renouvelé.

Si un processus d'interprétation appelle des choix singuliers, du même ordre que ceux que génère la traduction, le texte d'action est néanmoins très contraignant et la notation obéit au principe de fidélité à sa source. Nous nous situons dans une démarche essentiellement «top-down ». 
A partir des années 1980, des théoriciens du mouvement comme Ann Hutchinson Guest $^{7}$ à Londres ont conçu un système de notation simplifié, appelé « Motif Notation », qui invite les danseurs à explorer tous les possibles à partir de signes, donc à improviser à partir d'un code commun. Les signes sont délibérément polysémiques, de telle sorte que chaque danseur incarne chaque unité d'action à sa manière.

\section{PARTNERS RELATING (Ending Together)}

A duet for boy and girl telling a little story.

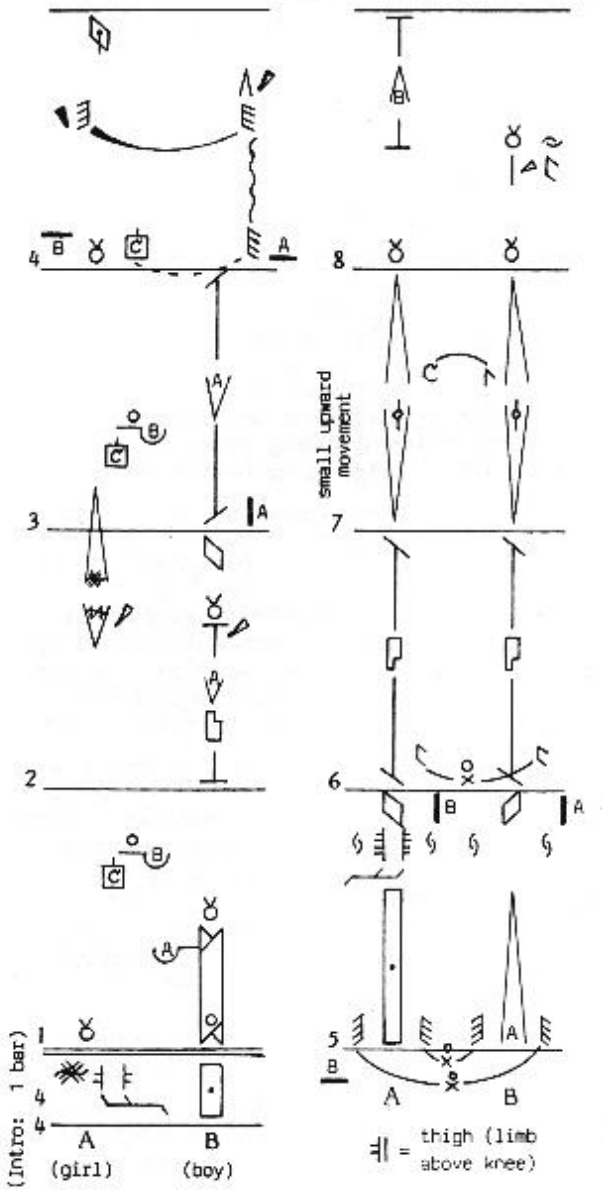

Exemple de partition de "Motif Notation». (Guest 148). Il s'agit ici de la trame d'un duo. Deux lignes verticales parallèles, lues de bas en haut, symbolisent le texte d'action de chacun des deux partenaires. Si les signes indiquent bien à chaque danseur le type d'action qu'il doit réaliser (ex : au début du duo, (B) doit s'adresser à (A) par une torsion du corps), rien n'est précisé quant à la manière dont l'action doit être exécutée : (B) aura-t-il recours à une torsion vers la gauche? Vers la droite ? Se contentera-t-il de regarder sa partenaire ou engagera-t-il tout le buste?

La performance émerge donc de la créativité de chacun et du hasard des trouvailles à chaque nouvelle improvisation, mais le spectateur qui assiste à deux performances issues du même texte d'action ressent qu'un code commun unit les danseurs. Si interpréter une partition chorégraphique obéit plutôt à un mouvement «top-down», improviser à partir 
d'une partition de « Motif Notation » privilégie une dynamique «bottom-up », caractéristique d'une démarche inclusive.

\section{Contribuer à la création d'une performance, c'est se sentir compétent}

L'ensemble des processus créatifs dont il a été question obéit au même principe : la diversité est constitutive de l'humain. Elle est une richesse en soi. Toute dynamique inclusive partira donc de ce que peut apporter chacun à l'action collective, posée comme une cocréation, dont le résultat, par essence imprévisible, appartient au complexe en raison de la diversité des stratégies mises en œuvre et de l'alchimie que constitue leur combinatoire. Dans de tels espaces, au propre et au figuré, les acteurs se sentent compétents, car le projet coopératif se construit à partir des trajectoires singulières, sans cesse revisitées pour optimiser l'action commune.

\section{Vers une dynamique inclusive en classe de langue étrangère ?}

Publié en 2000, le Cadre Européen Commun de Référence pour les Langues (CECRL) pose l'usager / apprenant d'une langue comme un «acteur social», couplant intimement usage de la langue et action :

L'usage d'une langue, y compris son apprentissage, comprend les actions accomplies par des gens qui, comme individus et comme acteurs sociaux, développent un ensemble de compétences générales et, notamment une compétence à communiquer langagièrement. [...] Les compétences sont l'ensemble des connaissances, des habiletés et des dispositions qui permettent d'agir. (15)

Nous avons vu précédemment que la performance appartient à l'action. Nous avons vu également que la performance en danse contemporaine, par le processus de création duquel elle résulte, privilégiant le mouvement «bottom-up » par rapport au «top-down », favorise l'inclusion. Ce processus est-il transférable à la classe de langue ? A quelles conditions ?

La clé nous parait se situer dans la conception même de la séquence d'enseignementapprentissage. Pour que l'apprenant d'une langue étrangère se sente compétent, il faut qu'il se sente « habile» dans son action mobilisant la langue étrangère. Dès lors, comment, pour l'enseignant, mettre l'élève en position d'apprivoiser l'étrangeté de manière à ne pas développer de sentiment d'exclusion?

Nous sommes confrontés ici, dans le domaine de l'enseignement-apprentissage d'une langue étrangère, à une rupture épistémologique de même nature que celle qui s'est opérée 
entre la notation classique du mouvement dansé et la «Motif Notation »: de même qu'il ne s'agit plus d'interpréter une partition chorégraphique en privilégiant une démarche «topdown », mais d'improviser dans une dynamique de création «bottom-up », la démarche actionnelle du CECRL invite le pédagogue en langues à inverser le mouvement posé par l'approche communicative. Il s'agit désormais pour l'apprenant de langue étrangère non plus seulement d'échanger des informations dans des situations données, mais d'être capable de contribuer en contexte social à des actions communes. S'atteler à une «tâche » avec d'autres n'est ni plus ni moins que réaliser un «texte d'action », ou performance, au sens défini précédemment.

Est définie comme tâche toute visée actionnelle que l'acteur se représente comme devant parvenir à un résultat donné en fonction d'un problème à résoudre, d'une obligation à remplir, d'un but qu'on s'est fixé. Il peut s'agir tout aussi bien, suivant cette définition, de déplacer une armoire, d'écrire un livre, d'emporter la décision dans la négociation d'un contrat, de faire une partie de cartes, de commander un repas dans un restaurant, de traduire un texte en langue étrangère ou de préparer en groupe un journal de classe. (16)

Tout comme les symboles notés sur une partition chorégraphique renvoient à des étapes d'une action finalisée, les tâches énumérées ci-dessus passent par des étapes, ou unités d'action. Dans le cadre d'une séquence d'enseignement-apprentissage conçue dans une perspective actionnelle, les didacticiens parlent souvent de «tâches intermédiaires » menant à une « tâche finale ».

Les tâches finales sont généralement complexes ${ }^{8}$ :

- il n'y a pas de solution idéale pour les réaliser ;

- il faut tâtonner pour résoudre les problèmes rencontrés, en s'appuyant sur des repères jugés pertinents ;

- ces repères et les stratégies mises en œuvre sont variables selon les personnes ;

- les interactions génèrent des incertitudes et de l'imprévu auxquels chaque acteur doit s'adapter ;

- plusieurs solutions peuvent être jugées satisfaisantes pourvu qu'elles mobilisent de manière articulée et efficace des ressources appropriées.

Confronter les élèves en situation d'apprentissage à une telle complexité est-il réaliste ? Le risque n'est-il pas de renforcer chez l'élève le sentiment d'inaccessibilité des compétences travaillées qui génère la souffrance évoquée plus haut?

L'expérience pédagogique montre que c'est moins la nature complexe de la tâche qui met l'élève en difficulté que la démarche adoptée pour la résoudre. Face à la complexité, le 
réflexe observable chez de nombreux enseignants est de tenter de compenser par un guidage fort, répondant à une démarche simplificatrice, organisée selon un mouvement «top-down ». L'élève y est mis en position d'exécutant servile selon des stratégies qui lui sont imposées et ne font pas nécessairement sens pour lui.

Or le complexe ne se laisse pas réduire à une série d'opérations simples pouvant être dictées; il implique pour chaque acteur de contribuer à la tâche commune, non pas avec simplicité, mais avec «simplexité ». Selon A. Berthoz, cette notion est très proche de l'intuition qui guide nos actes dans des environnements complexes (224). Le mouvement «bottom-up » y prédomine.

\section{Du complexe au simplexe}

«Devant la complexité du monde, le vivant trouve des solutions d'une exceptionnelle élégance », dit Berthoz (104) : «Un cerveau humain est créateur de mondes. [...] Un tel mode de fonctionnement est essentiellement simplexe, car il affranchit notre machine cérébrale de la réalité complexe du monde en lui permettant de naviguer dans [une] simulation virtuelle $\gg(221)$.

Dans l'approche communicative de l'enseignement-apprentissage des langues étrangères, il était essentiellement question pour les apprenants de savoir échanger des informations dans des situations données. Le format d'enseignement consistait donc à poser des situations d'énonciation et à attribuer des rôles aux élèves de telle sorte qu'ils puissent produire par imitation des échanges à l'intérieur de ces cadres, en suivant pas à pas des consignes strictes ${ }^{9}$.

Avec l'avènement de la perspective actionnelle, il s'agit dorénavant de poser des tâches complexes à la réalisation desquelles chaque apprenant doit pouvoir contribuer avec simplexité. Il n'incombe plus aux élèves d'appliquer une démarche toute faite, mais il appartient à chaque élève de proposer sa stratégie, à confronter à celle de ses pairs. Un tel processus pédagogique, où le mouvement «bottom-up » prédomine, rencontre la notion de «zone proximale de développement » définie par Vygotski en 1934 : «La possibilité plus ou moins grande qu'a l'enfant de passer de ce qu'il sait faire tout seul à ce qu'il sait faire avec quelqu'un est précisément le symptôme le plus notable qui caractérise la dynamique de son développement et de la réussite de son activité intellectuelle. Elle coïncide entièrement avec sa zone proximale de développement » (271). 
Pour terminer notre parallèle avec la danse contemporaine, prenons un exemple de tâche finale complexe posée en classe de seconde en anglais : «Créez et jouez un scénario qui fasse comprendre à un public comment fonctionne le système des temps de la narration au passé en anglais ».

Les tâches intermédiaires proposées pour mener à bien cette séquence ont suivi les étapes de la construction d'une performance en danse contemporaine :

\section{Tâche 1 (Mise en jeu de la créativité par des inducteurs)}

En binômes, imaginez une brève histoire sans paroles (mime ou dessin). Présentez-la à la classe.

\section{Tâche 2 (Improvisation / exploration)}

Choisissez l'histoire d'un autre binôme et racontez-la par écrit, au passé, d'abord de manière simple. Puis étoffez l'histoire et utilisez au moins deux temps différents du passé (ceci donnera lieu à un travail de révision des temps, des mots de liaison et des phrases complexes en classe entière à partir d'exemples). Réalisez une carte d'identité pour chaque temps du passé et présentez-la à la classe.

\section{Tâche 3 (Composition)}

En petits groupes, créez un scénario réalisant la tâche finale. Vous pouvez repartir de votre histoire précédente ou en inventer une autre. Vous pouvez vous inspirer d'histoires mises à votre disposition (banque de supports textes, audio, vidéo). L'écriture de votre scénario sera évaluée (créativité, pertinence, correction).

\section{Tâche 4 (Production)}

Travaillez le jeu scénique en ateliers en alternance avec des entrainements individuels (TICE, assistant).

\section{Tâche 5 (Présentation)}

Jouez et évaluez la performance des autres groupes selon des critères dont vous conviendrez.

Comme dans le cas de la création d'une performance en danse contemporaine, la séquence est sous-tendue par une dynamique inclusive, où chaque élève trouve sa place, grâce à un mouvement essentiellement «bottom-up », même si des phases «top-down » sont posées par l'enseignant pour apporter des connaissances ou systématiser certains entrainements :

- les tâches renvoient à l'action créative, avec des verbes comme «inventer », «créer », « réaliser $» . .$. ;

- chaque élève est co-auteur et co-acteur du projet ; 
- à partir de propositions hétérogènes, des regroupements s'opèrent pour donner naissance à des ateliers de création autonomes ;

- chaque atelier propose un texte d'action différent, réalisation singulière de la tâche finale énoncée au départ ;

- chaque élève, aidé par l'enseignant, définit et assume son rôle propre à l'intérieur d'un groupe ;

- l'enseignant est là pour donner les matériaux nécessaires lorsqu'un obstacle cognitif / langagier surgit. Certains besoins sont communs à tous les élèves et les apports qu'ils appellent pour être comblés peuvent être gérés en classe entière ; d'autres nécessitent des interventions adaptées selon les groupes ou les individus ;

- chaque élève peut progresser par rapport à lui-même, à l'intérieur de sa «zone proximale de développement ». Il est encouragé par l'enseignant et par ses pairs à pousser plus loin l'exploration sous forme d'improvisations diverses ;
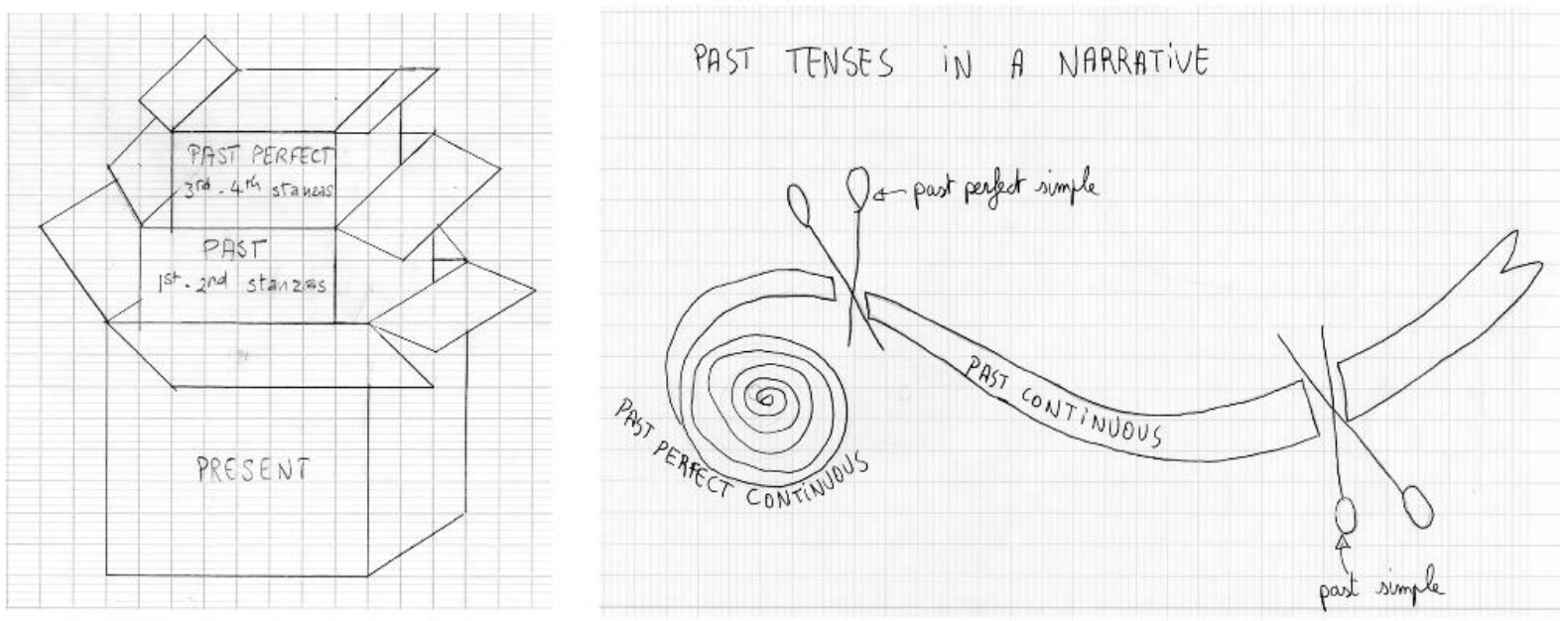

Exemples de représentations des temps du passé pour la carte d'identité demandée en tâche 2 .

- l'évaluation s'organise autour de critères que se donne le groupe, mais aussi autour de critères que choisit chaque élève selon ses points forts et les objectifs d'apprentissage qu'il se pose, aidé par l'enseignant.

\section{En conclusion, que pourrait être le «présent d'incarnation » en classe de langue ?}

« Ça y est! Mon cerveau diffuse dans mon corps : ça s'incarne. », nous dit Pennac. Sans doute est-ce là ce que nous ressentons lorsque, dans la vie, nous nous sentons compétents pour agir dans une situation complexe. Se sentir compétent, c'est ressentir que 
notre être tout entier est capable de trouver les gestes et les paroles qui nous permettent d'agir de manière simplexe :

Un aspect fondamental de la simplexité est cette activité créatrice de notre cerveau. Celui-ci résout le problème de la complexité du monde extérieur en produisant des perceptions compatibles à la fois à ses intentions pour le futur, sa mémoire du passé et les lois du monde extérieur qu'il a internalisées. Il crée, au fond, un véritable Umwelt. Ce qui a un prix : l'erreur. (Berthoz 78)

Le présent d'incarnation en classe de langue?

Un temps où le corps propre de chaque élève, véhicule de ses émotions, peut s'exprimer sans crainte de la faute car porté par un projet qui le met en mouvement, tolérant l'erreur inhérente à toute activité de conception.

Un espace où le sentiment d'appartenir à un corps social élargi incite à l'apprentissage et à la co-action.

Un art d'enseigner / d'apprendre dans une esthétique du divers, fondement même d'une dynamique d'inclusion : «Si les arts ont prévalu au cours de l'évolution, c'est parce qu'ils avaient une valeur pour la survie et ont contribué au développement de la notion de bien-être » (Damasio 359).

Car finalement, quelle que soit la compétence concernée, «pour acquérir une dimension proprement artistique, le savoir-faire doit se conjuguer à de 'l'amour'. » (Dewey 100). Aimer, pour un élève, c'est se sentir inclus dans cet espace-temps, cet art de l'apprentissage, de l'Autre, où «ça s'incarne » dans un corps propre et social vivant.

\section{Bibliographie}

Ancet, P. et D. Toubert-Duffort, Dirs. «Corps, identité, handicap ». La nouvelle revue de l'adaptation et de la scolarisation 50 (2010).

Berthoz, A. La simplexité. Paris : Odile Jacob, 2009.

Bodak, S. La danse de Malkovsky à travers la notation Laban. Ressouvenances, 2009 (2001).

Crémézi, S. La signature de la danse contemporaine. Paris : Chiron, 1997.

Conseil de l'Europe. Cadre européen commun de référence pour les langues. Paris : Didier, 2000.

Cyrulnik, B. Mourir de dire. La honte. Paris : Odile Jacob, 2010.

Damasio, A. L'autre moi-même. Les nouvelles cartes du cerveau, de la conscience et des émotions. Paris : Odile Jacob, 2010.

Dewey, J. L'art comme expérience. Paris : Folio essais, 2010 (1915). 
Hecquet, S. et S. Prokhoris. Fabriques de la danse. Paris : PUF, 2007.

Hutchinson Guest, A. Your Move. A New Approach to the Study of Movement and Dance. Amsterdam : Gordon \& Breach, 1983.

Lecoq, J. Le corps poétique : un enseignement de la création théâtrale. Paris : Actes Sud, 1997.

Pennac, D. Chagrin d'école. Paris : Gallimard, 2007.

Perez, T. et A. Thomas. Danser les arts. Nantes : CRDP des Pays de la Loire, 2000.

Schechner, R. 2002. Performance Studies. Londres et New York : Routledge, 2002.

Vygotski, L. Pensée et Langage. Éditions Sociales, 1985.

\section{NOTES}

${ }^{1}$ Adaptation scolaire et Scolarisation des élèves en situation de Handicap.

${ }^{2}$ «Performance » s'entend tout au long de cet article dans son acception anglo-saxonne.

${ }^{3}$ Pour avoir un aperçu du travail de la chorégraphe angevine, Marie-France Roy, voir le site de l'Association Résonnance - Angers. http://www.resonnancedanse.com/Resonnance/asso.html (consulté le 20 juillet 2012).

${ }^{4}$ Rizzolatti, G. et C. Sinigaglia. Les Neurones Miroirs. Paris : Odile Jacob, 2008.

${ }^{5}$ Un processus «bottom-up » part des propositions singulières des danseurs, mises en synergie par le chorégraphe; un processus «top-down» met les danseurs en situation de reproduction-interprétation des propositions du chorégraphe

${ }^{6}$ "Inclusion is more than a set of strategies or practices, it is an educational orientation that embraces differences and values the uniqueness that each learner brings to the classroom. " http://www.paulakluth.com/readings/inclusive-schooling/is-your-school-inclusive/ (consulté le 22 juillet 2012, notre traduction)

${ }^{7}$ J'adresse ici tous mes remerciements à Ann Hutchinson Guest pour ses précieuses indications concernant l'analyse de la partition dont elle est l'auteur.

${ }^{8}$ Gérard, F.-M. 2008. La complexité d'une évaluation des compétences à travers des situations complexes : nécessités théoriques et exigences de terrain. http://www.fmgerard.be/textes/complexeval.html (consulté le 22 juillet 2012).

${ }_{9}^{9}$ Puren, C. 2008. Formes pratiques de combinaison entre perspective actionnelle et approche communicative : analyse comparative de trois manuels. http://www.aplv-languesmodernes.org/spip.php?article1409 (consulté le 23 juillet 2012). 